\title{
CRISIS ECONÓMICAY \\ MIGRACIÓN LABORAL INTERNACIONAL: COSTOS Y BENEFICIOS EN ALEMANIA
}

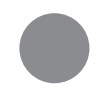

\author{
BÉATRICE KNERR
}

TRADUCCIÓN DE LUIS RODOLFO MORÁN QUIROZ

RESUMEN. Alemania se ha tornado gradualmente en un país de emigración. Desde principios de los años noventa se encuentra en una crisis económica, y el desempleo se ha convertido en un fenómeno masivo. Tasas de desempleo de más del 10\%, condiciones de trabajo cada vez más deterioradas y poca esperanza de mejoría inducen a una cifra creciente de alemanes, y de extranjeros previamente inmigrados, a buscar mejores oportunidades más allá de las fronteras alemanas. Al mismo tiempo, decae la cifra de los inmigrantes. Las reacciones ante este nuevo fenómeno son ambiguas, aunque sus consecuencias apenas se investigan. La falta de datos y de información en torno a los parámetros conductuales dificulta el análisis de sus costos y beneficios. Aunque si se le mide por la proporción de la mano de obra total, la emigración todavía no parece tan alarmante, y podría considerarse como un alivio para el mercado de trabajo y para la hacienda pública, sí es posible señalar que su composición y perspectivas parecen amenazar cada vez más la economía alemana, pues los altos potenciales, cada vez más escasos, muestran una propensión exagerada en términos proporcionales a emigrar e imponen altos costos de oportunidad sobre la economía y la sociedad. Además, la experiencia internacional sugiere que debido a las redes sociales, el goteo de la emigración puede rápidamente convertirse en un río sostenido por su misma fuerza. Este flujo de salida no se ve equilibrado por un flujo de ingreso adecuado de mano de obra calificada y los sectores clave pueden enfrentar una escasez de fuerza de trabajo competente que se convierta en un lastre para el nuevo ímpetu que requiere la economía de Alemania.

palabras ClaVe. Migración, Alemania, mercado de trabajo, análisis costo-beneficio, crisis económica.

ABSTRACT. Germany which for decades has been known as a country of large-scale immigration of labour force is increasingly turning into a country of out-migration. Since the early 1990s, it finds itself in an economic crisis, and unemployment has turned into a mass phenomenon, seizing almost all skill and educational levels. Unemployment rates of more than $10 \%$, worsening working conditions, and little hope for improvement induce an increasing number of Germans and previously immigrated foreigners to look for better chances beyond Germany's borders. Concomitantly, the number of in-migrants declines. The reactions to this new phenomenon are mixed, although its consequences are hardly investigated and not clear to a broader public. Lack of data and information about behavioural parameters make an analysis of its costs and benefits difficult. Although as measured by the share of the total labour force, out-migration still does not appear alarming and might appear as a relief to the labour market and the public treasury, its composition and perspectives increasingly seem to threaten Germany's economy, as high-potentials who are increasingly scarce, display an over proportional propensity to migrate and impose high opportunity costs on economy and society. International experience moreover, suggests that due to networking, the trickle of out-migration may rapidly develop into a self-sustaining stream. This outflow is not counterbalanced by an adequate inflow of qualified labour force, and key sectors might face a lack of competent labour force which might already have become a handicap for the new fresh impetus that the German economy needs.

KEYWORDs. Migration, Germany, labour market, cost benefit analysis, economic crisis. 


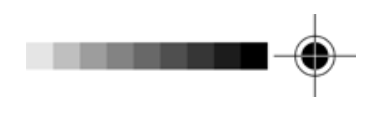

\section{INTRODUCCIÓN}

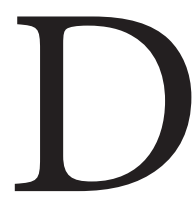

urante siglos Alemania ha sido un país de inmigración de mano de obra a gran escala. De los años cincuenta a los setenta, un periodo de expansión económica y cada vez más escasez de mano de obra, en particular en el segmento de la mano de obra poco calificada, el país puso en marcha un «programa de trabajadores huéspedes» que, durante sus primeros años, atrajo al país a cientos de miles de trabajadores del sur de Europa, principalmente de Italia, España y Portugal, más tarde de Turquía y de Europa del Este. Al mismo tiempo, Alemania se convirtió en una de las más grandes fuentes de remesas en el mundo.

Hasta el inicio de los años ochenta, la mano de obra extranjera, como complemento del capital en la producción, aceleró el crecimiento económico alemán. Desde la segunda mitad de esa década, cuando las tasas de crecimiento ya no fueron tan altas, esta misma mano de obra comenzó a ser percibida como una carga para los sistemas de seguridad sociales del país. Aunque el gobierno intentó evitar la llegada de mano de obra adicional y convencer a quienes quedaban desempleados de que regresaran a sus países de origen, siguió creciendo la cifra de extranjeros que buscaban empleo en Alemania.

En los años noventa se invirtió al fin la tendencia: con la reunificación de Alemania occidental con su parte oriental - la antigua República Democrática Alemana- y bajo la presión de una globalización económica creciente, el país entró en una crisis económica. El desempleo se convirtió en un fenómeno masivo y ha afectado a casi todos los niveles de preparación y escolaridad de la fuerza laboral. El país ha tenido que acostumbrarse a tasas permanentes de desempleo de más del $10 \%$ y a salarios reales decrecientes. Una primera consecuencia fue la disminución en el flujo de llegada de mano de obra, lo que fue apoyado por los esfuerzos del gobierno por proteger el mercado laboral nacional. Ello seguido de una creciente emigración de la fuerza laboral extranjera desde Alemania.

En esas circunstancias, un número creciente de alemanes busca mejores oportunidades fuera de las fronteras del país. Las reacciones ante este nuevo fenómeno son ambiguas, aunque sus consecuencias apenas han sido investigadas y en gran parte no quedan claras para el público más amplio (Bundesamt für Migration und Flüchtlinge, 2006). Además, la inmigración ha descendido y un número creciente de extranjeros regresa a sus países de origen, o bien, dejan Alemania hacia un tercer país.

En la comunidad científica, la emigración desde Alemania es un campo desatendido por la investigación. (Bundesamt für Migration und Flüchtlinge, 2006: 114). No obstante, este fenómeno recibe creciente atención en los medios de comunicación abiertos. En particular la emigración de la mano de obra altamente calificada atrae el interés del público en el contexto de la «fuga de cerebros».

En este artículo exploro las consecuencias económicas de la salida de mano de obra desde Alemania, a partir del análisis de costo-beneficio. Comienzo con la hipótesis de que la crisis económica de Alemania causa movimientos internacionales de mano de obra que implican, para la economía alemana, costos más altos que beneficios y que, en el largo 
plazo, pueden limitar el crecimiento económico. Con ese propósito estudio por separado la emigración de la fuerza de trabajo altamente calificada, de los trabajadores manuales calificados y de los trabajadores de escasa capacitación, además, la migración de retorno y la migración de segundo paso de la mano de obra extranjera. Al hacerlo, considero los aspectos de los costos de oportunidad de la fuerza de trabajo perdida, las remesas y el posible reflujo del capital humano adquirido en el extranjero. En correspondencia con la debilidad de los datos disponibles, las demandas cuantitativas tuvieron que ser moderadas.

Este artículo se estructura de la siguiente manera: la sección dos explica el desarrollo de la emigración de Alemania, tomando como telón de fondo el crecimiento económico del país. La sección tres presenta los cálculos de costo-beneficio y la sección cuatro presenta las conclusiones.

\section{EMIGRACIÓN DESDE ALEMANIA}

\section{Desarrollo económico y mercado laboral}

Durante siglos, Alemania fue un motor de crecimiento económico dentro de Europa. Durante los años sesenta y setenta del siglo pasado, a pesar de las tasas positivas de crecimiento de la mano de obra nacional, el país atrajo grandes cantidades de trabajadores de los países del sur de Europa y, más tarde, de Turquía, por medio de programas de reclutamiento activo (Castles, 1986; Straubhaar, 1988; Körner, 1990; Knerr 1997).

\section{FIGURA 1}

Crecimiento económico y tasas de desempleo en Alemania, 1970-2004

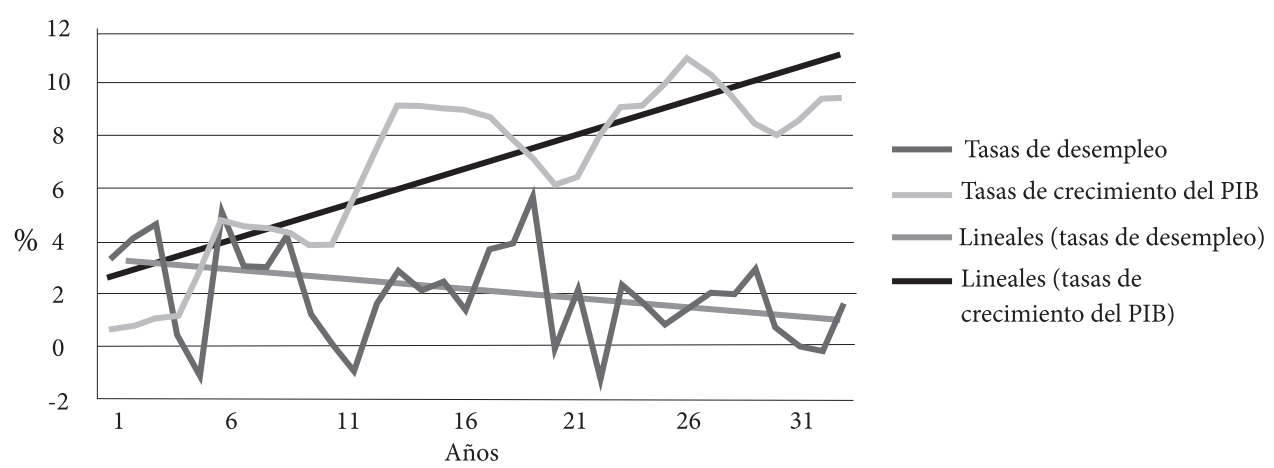

Fuente: elaborado con base en datos de la Statistisches Bundesamt Deutschland y WSI (2005) (datos de la Comisión de la UE).

Desde principios de los años noventa, el crecimiento económico nacional ha seguido una tendencia a la baja y las tasas de desempleo alcanzaron niveles sin precedente, reaccionado a las tasas de crecimiento con una diferencia temporal de hasta dos años 
(figura 1). Al mismo tiempo, bajaron los salarios reales, lo que convirtió a Alemania en el único país dentro de la Unión Europea (UE) que mostró un descenso en los salarios reales (Claus 2006). En la década de 1995 a 2004 bajaron en 0.9\%, mientras que en el mismo periodo, dentro de la Unión Europea (UE) alcanzaron un aumento promedio de $7.7 \%$. En la parte más alta se situaban los estados miembros de Suecia y Gran Bretaña, lugares en los que los salarios reales crecieron más del 25\%, mientras que en Estados Unidos se incrementaron en casi $20 \%$.

Aún cuando las tasas de salarios en Alemania no se sitúan en un nivel bajo, si se comparan con las de otros estados miembros - y son todavía mejores en comparación con parámetros internacionales- las diferencias positivas se reducen cada vez más. Los impactos que ha sentido la fuerza laboral se dan en particular en la inseguridad en el empleo, reducción en los beneficios por desempleo y peores condiciones laborales, como más horas de trabajo, menos prestaciones y generalmente una presión creciente. De tal modo que en menos de una década, la proporción de contratos de trabajo limitados en términos de horarios se duplicó para los jóvenes de menos de 25 años y se incrementó en más de un tercio para el rango de edad entre 25 y 35 (tabla 1).

TABLA 1

Porcentaje de contratos por tiempo limitado, 1995 y 2003

\begin{tabular}{|c|c|c|}
\hline RANGO DE EDAD & 1995 & 2003 \\
\hline $15-20$ & 31.5 & 80.9 \\
\hline $21-25$ & 20.9 & 43.2 \\
\hline $26-30$ & 10.2 & 16.6 \\
\hline $31-31$ & 5.6 & 8.3 \\
\hline
\end{tabular}

Fuente: basado en datos de la Statistisches Bundesamt, serie 1; cit. en Köhne.

La demanda decreciente de mano de obra fue seguida de una oferta subsidiada de mano de obra expatriada, la creciente migración de retorno o migración de segundo paso de los extranjeros y - un nuevo fenómeno en Alemania- la emigración a gran escala de la fuerza de trabajo alemana.

\section{Migración cambiante por encima de las fronteras}

Desde los años noventa, la distancia entre la inmigración y la emigración se ha reducido significativamente (fig. 2). ${ }^{1}$ En particular, el número de inmigrantes ha bajado conside-

\footnotetext{
${ }^{1}$ Las cifras oficiales no incluyen a la gran cantidad de personas de origen étnico alemán (los llamados Spätaussiedler) que inmigran de Europa del este (casi exclusivamente provenientes de la antigua Unión Soviética), quienes reciben la ciudadanía alemana en cuanto cruzan la frontera germana. Su cifra llegaba a 397,073 personas en 1990 y luego decayó, de manera estable, a 59,093 en 2004 (Migrationsbericht, 2006: 46)
} 
rablemente durante el periodo 1991-2005. En 2004, Alemania registró el número más bajo de inmigrantes durante más de una década. Además, un número creciente de alemanes, los antiguos inmigrantes, así como los migrantes de segunda generación salen de Alemania en busca de empleo en el extranjero, regresar a su país de origen, al país de sus padres o hacia un tercer país. Tan sólo entre 2003 y 2004, la cantidad de extranjeros en el país disminuyó en 8.4\% (Migrationsbericht 2006: 117). Como consecuencia, en 2005, por primera vez, más personas salieron del país de las que llegaron a él.

\section{FIGURA 2}

Migración hacia y desde Alemania, 1991-2004

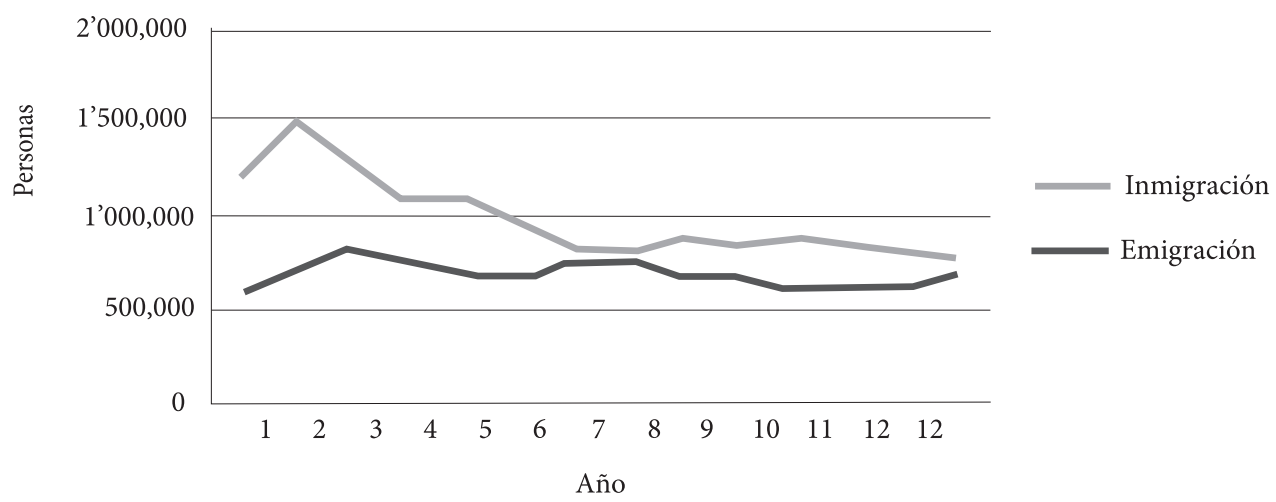

Fuente: basado en datos de la Bundesamt für Migration und Flüchtlinge, 2006.

La relación entre la migración y el desarrollo económico de Alemania queda más clara por los movimientos migratorios entre Alemania y sus 14 socios de la UE. Sus etapas de desarrollo económico están cercanas a la de Alemania y, por tanto, se puede observar que las migraciones transfronterizas reaccionan sensiblemente a las diferencias cambiantes en los parámetros económicos entre estos países. Desde los años noventa, la migración entre Alemania y sus catorce socios de la UE, ${ }^{2}$ a excepción de Holanda y el caso especial de Luxemburgo, cambió de positiva a negativa (fig. 3) junto a los salarios reales descendentes en Alemania y crecientes salarios reales en los otros 14 países de la uE (tabla 2).

Desde 1996, el saldo migratorio entre Alemania y los 14 países de la UE, es decir, frente a los estados miembros más ricos, es negativo. De un total de +27,487 en 1991, bajó a menos 43,705 en 2004 (fig. 4). En contraste con ello, ha sido positivo frente a los nuevos (y más pobres) estados miembros de la $\mathrm{UE}^{3}$ (con base en datos de la Stat. Bundesamt, 2006) y llegaron a 36,634 personas en 2004. Tan sólo Polonia mostró un saldo de $+27,236$ personas, que es cerca del doble del año anterior.

\footnotetext{
${ }^{2}$ Se trata, esencialmente, de países industrializados en el sur y oeste de Europa: Bélgica Dinamarca, Finlandia, Francia, Grecia, Irlanda, Italia, Luxemburgo, Holanda, Austria, Portugal, Suecia, España, Reino Unido.

${ }^{3}$ Países del centro y del este de Europa, que se unieron a la UE en 2004.
} 
FIGURA 3

Migración transfronteriza desde Alemania a los 14 miembros de la UE

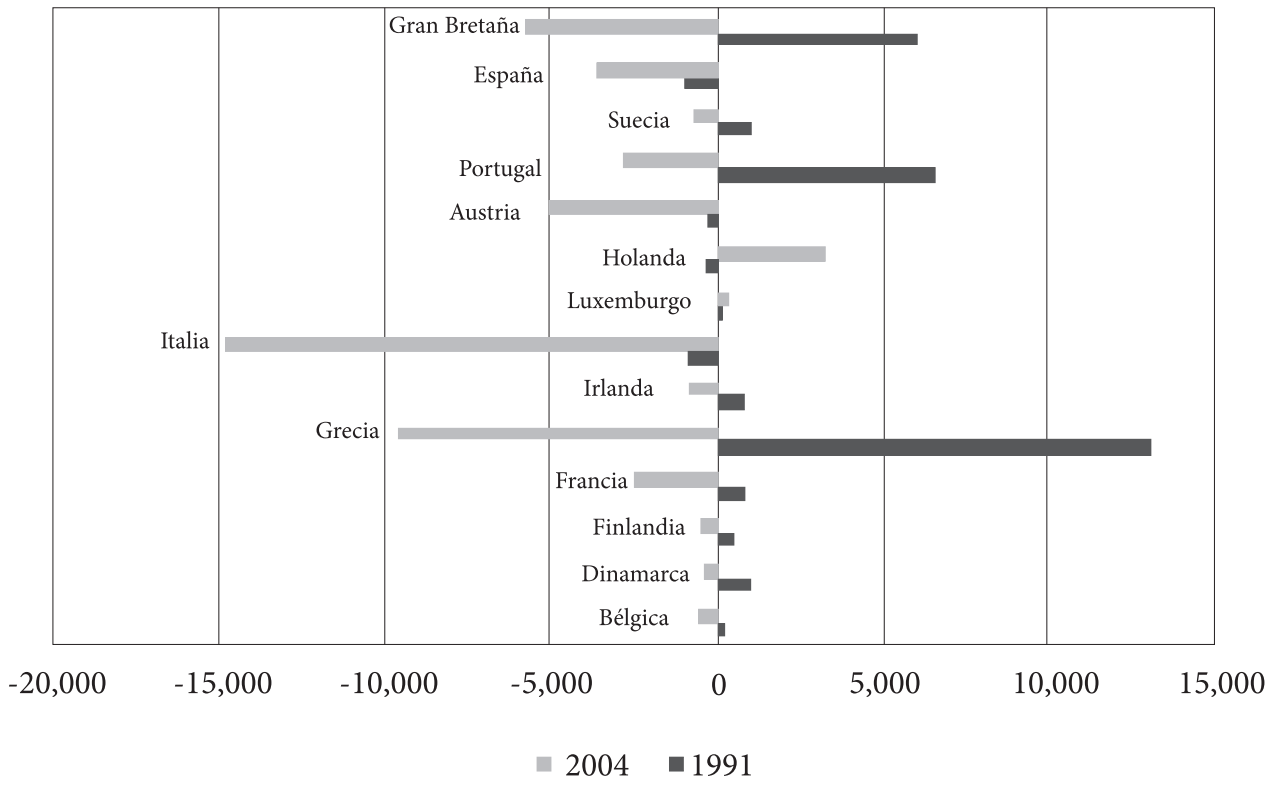

Fuente: basado en datos de la Statistisches Bundesamt.

FIGURA 4

Migración de ciudadanos de los 14-Ue hacia y desde Alemania, 1991-2004

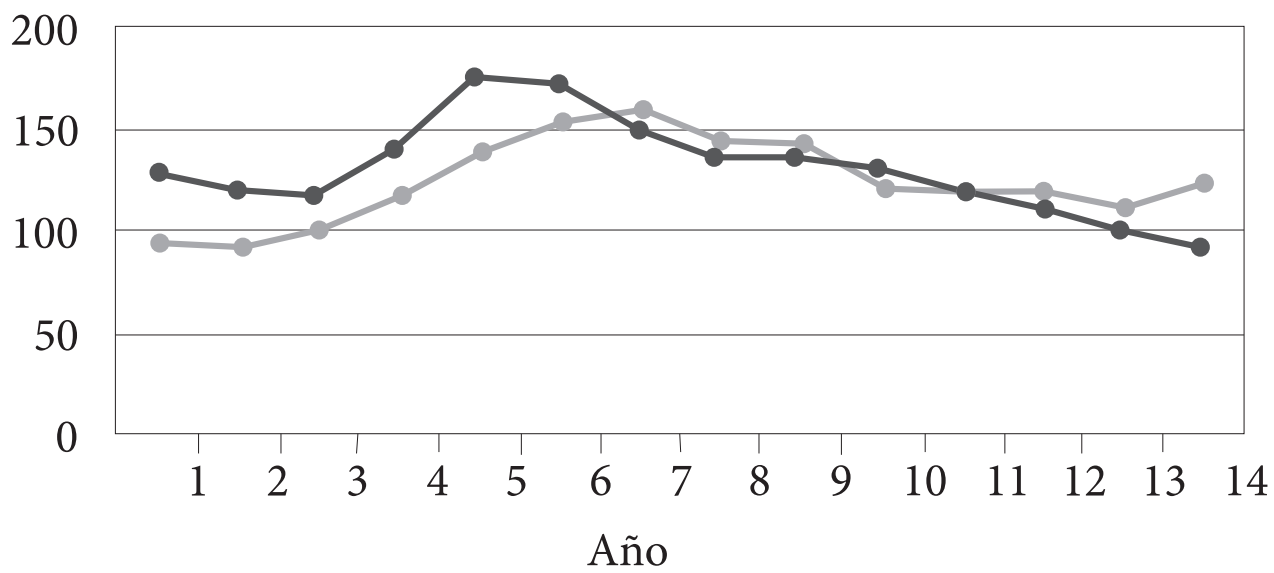

\section{Emigración — Inmigración}

Fuente: basado en datos de la Statistisches Bundesamt. 
TABLA 2

Desarrollo de los salarios reales y emigración desde Alemania

\begin{tabular}{|c|c|c|c|c|c|}
\hline PAÍS DE DESTINO & $\begin{array}{c}\text { MIGRACIÓN } \\
\text { NETA } 1995\end{array}$ & $\begin{array}{l}\text { MIGRACIÓN } \\
\text { NETA } 2004\end{array}$ & $\begin{array}{c}\text { INCREMENTO EN } \\
\text { SALARIOS REALES } \\
\text { 1995-2004 }\end{array}$ & $\begin{array}{c}\text { DIFERENCIA PARA } \\
\text { EL INCREMENTO } \\
\text { DE LOS SALARIOS } \\
\text { REALES ALEMANES }\end{array}$ & $\begin{array}{c}\text { \% INCREMENTO } \\
\text { EN LA } \\
\text { MIGRACIÓN } \\
\text { NETA }\end{array}$ \\
\hline Austria & 13 & $-5,062$ & 2.8 & 3.7 & $-39,038$ \\
\hline Holanda & 1,273 & 3,245 & 11.9 & 12.8 & 155 \\
\hline Bélgica & -246 & -587 & 6.4 & 7.3 & -136 \\
\hline Francia & 1,315 & $-2,477$ & 8.4 & 9.3 & -288 \\
\hline Suecia & 698 & -684 & 25.4 & 26.3 & -198 \\
\hline Gran Bretaña & 1,289 & $-5,810$ & 25.2 & 26.1 & -550 \\
\hline Irlanda & 529 & -834 & 19.4 & 20.3 & -258 \\
\hline Italia & 15,349 & $-14,851$ & 2.0 & 2.9 & -196 \\
\hline España & $-1,269$ & $-3,604$ & 5.4 & 6.3 & -184 \\
\hline Dinamarca & -314 & -384 & 16.6 & 17.5 & -22 \\
\hline Grecia & 1,126 & -934 & n.a. & - & -183 \\
\hline Portugal & 10,838 & $-2,873$ & n.a. & - & -126 \\
\hline
\end{tabular}

Fuente: basada en datos de wsi, 2005, y la Statistisches Bundesamt, 2006

Además, la porción más considerable de la población de migrantes en Alemania proviene de países con fuerzas de trabajo con un nivel de capacitación relativamente bajo. La mayor parte de estos inmigrantes permanecen, a pesar de la deteriorada situación del mercado laboral; algunos se van a un tercer país.

Al mismo tiempo, una parte significativa de la segunda generación, hijos de inmigrantes, se convirtió en un desafío para la economía. En contra de las expectativas anteriores, su integración en el mercado laboral alemán y a la sociedad —lo cual en buena parte se realizaría de manera coordinada - ha resultado difícil. Con baja escolaridad y conocimiento deficiente del idioma alemán, muchos de ellos no han podido acceder al mercado de trabajo. En cambio, contribuyen de manera desproporcionada al desempleo juvenil masivo y, como consecuencia, a tensiones sociales ampliamente difundidas. ${ }^{4}$

Resulta revelador tomar a la inmigración como consecuencia y como indicador de desarrollo económico y comparar la inmigración a Alemania con la que se da a otros países de la UE con tasas más altas de crecimiento en su producto interno bruto (РIB). Mientras que a principios de los años noventa la inmigración hacia Alemania ha decaído de manera constante, la inmigración a España e Italia, en el mismo periodo, se ha incrementado fuertemente, convirtiéndolos en importantes países receptores de migrantes. En 2004, por ejemplo, migraron a España 685,000 personas, mientras que

\footnotetext{
${ }^{5}$ Una buena parte de los migrantes de segunda generación, es decir, hijos de migrantes, nacidos y educados en Alemania, hablan incluso peor alemán que sus padres, lo que les pone en desventaja en la escuela.
} 
en 1999 habían sido tan sólo 127,000, es decir, se quintuplicó el número en cinco años. En Italia, la cantidad de inmigrantes se duplicó entre 2002 y 2003 para llegar a 445,000 personas. Procesos similares se han observado en Gran Bretaña, Austria y también en el nuevo estado miembro de la UE, la República Checa (Migrationsbericht, 2006). ${ }^{5}$

\section{Emigración de la fuerza de trabajo alemana}

La situación desfavorable del mercado laboral alemán en comparación con los países vecinos ha provocado que un número creciente de trabajadores, de escasa calificación y altamente capacitados por igual, abandone el país (Seidler, 2006). En 2004, más de 150,000 alemanes emigraron oficialmente, lo que representa un aumento del 18\% con respecto al año anterior y una cantidad mayor que en cualquier otro año desde 1950 (Statistisches Bundesamt, 2005). ${ }^{6}$ Por otro lado, durante los años setenta, la cantidad de emigrantes desde Alemania oscilaba entre las 50,000 y las 65,000 personas anualmente; en 1989, la cifra se elevó abruptamente a más de 100,000 anuales y desde entonces ha aumentado de manera casi uniforme (Bundesamt für Migration und Flüchtlinge, 2006: 114) (véase fig. 5). Este aumento en la emigración ha sido mucho más pronunciado que en cualquier otro país miembro de los 14-UE.

FIGURA 5

Emigración registrada desde Alemania por alemanes, 1991 a 2004

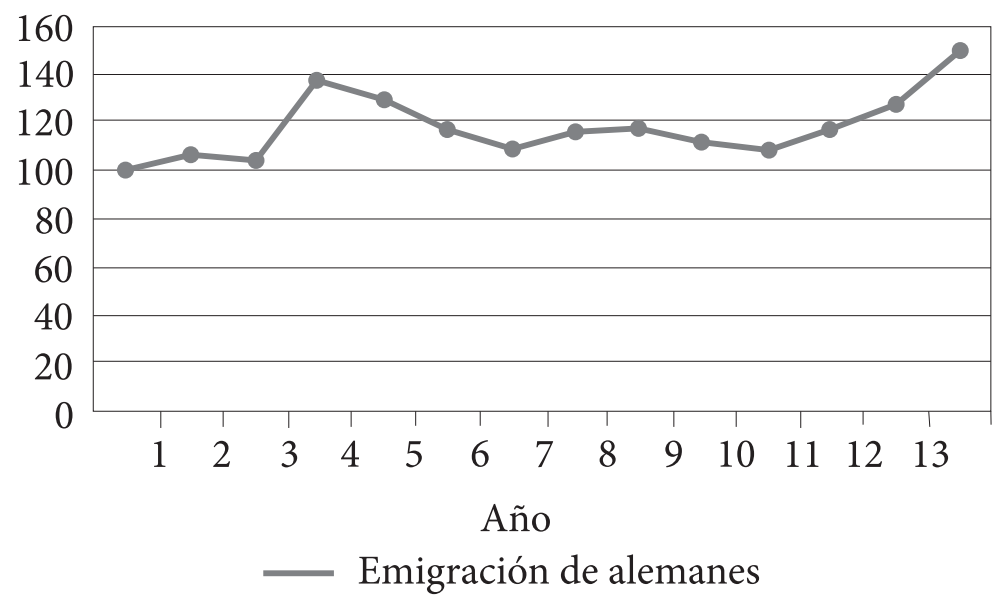

Fuente: basado en datos de la Bundesamt für Migration und Flüchtlinge y Statistisches Bundesamt, 2006.

\footnotetext{
${ }^{5} \mathrm{Al}$ comparar las cifras absolutas hay que tomar en cuenta que difieren las definiciones de los migrantes.

${ }^{6}$ Según las estimaciones de la revista económica Manager-magazin, con base en los datos disponibles del servicio alemán federal de estadística, esta cifra se incrementó a 160,000 en 2005, lo que significa un incremento del 8\% frente a los nueve meses incluidos para el periodo de 2004 (Müller, 2006b).
} 
Estas cifras incluyen solamente a quienes registran oficialmente su salida. Queda abierto a la especulación cuántos se han ido sin registrarse o viajan entre dos países (Seith 2006). Müller, de acuerdo con sus consultas a expertos, calcula que la cantidad de emigrantes alemanes alcanzó muy probablemente las 250,000 personas en 2005 (Müller 2006b). Además, los datos están sesgados a la baja, por el hecho de que en épocas de creciente globalización muchos empleos para académicos con alta capacitación en el nivel de la alta gerencia son cubiertos por las grandes empresas multinacionales a escala transnacional. Estas personas tampoco aparecen en las estadísticas.

Mientras que en el pasado eran principalmente las personas de altos potenciales las que salían de Alemania en busca de nuevas experiencias y desafíos en el exterior, desde el inicio de la crisis económica cada vez más trabajadores manuales, capacitados y no, cubren puestos de trabajo más allá de las fronteras alemanas porque ya no encuentran oportunidades económicas en el país (Nina Seidel en Seith, 2006). Muchas de estas oportunidades están mediadas por las agencias de colocaciones del gobierno, las que, por ejemplo, juegan un papel activo al organizar intercambios de trabajos en el extranjero en los que se ofrecen cientos de puestos de trabajo, y también por parte de las agencias de empleo privadas que se han multiplicado desde mediados de los años noventa. Los dos tipos de agencias reportan un incremento sin precedentes en las solicitudes desde principios del siglo. Los países más codiciados como receptores en la lista de prioridades de quienes expresan su interés en un empleo en el extranjero son Suiza, Austria, Reino Unido, España y Estados Unidos (Beunink 2006).

El centro de empleos del gobierno federal de Alemania ${ }^{7}$ ( $\mathrm{ZAV}$ ) apoya activamente la emigración de la fuerza de trabajo. En particular para los desempleados a quienes intenta encontrarles colocación en el extranjero, por ejemplo en Noruega, Irlanda u Holanda. Entre 2000 y 2005, la cantidad de contratos de trabajo mediados por el zAV se quintuplicó. Tan sólo en 2003-2004 éstos aumentaron en 50\%. El centro calculaba que medió en unos 10,000 puestos en el extranjero para los alemanes en 2006, entre ellos una gran cantidad de trabajadores manuales calificados, como son los carpinteros o trabajadores de la construcción. Aunque la política explícita del gobierno alemán es apoyar la emigración de los desempleados, el centro ayuda también a quienes buscan otro empleo. La mayoría de quienes recibieron esta mediación del zAV se dirigieron a Austria, Suiza y Holanda. Una razón de importancia para esta selección de países es la gran demanda en la industria turística, en donde se prefiere a los alemanes en vez de otras nacionalidades, sobre todo por su manejo del alemán como lengua materna.

Como explicara el vocero del centro de empleo del gobierno federal de Alemania (ZAv) en diciembre de 2005, los alemanes están cada vez más motivados a trabajar en el extranjero y se encuentran con que hay un considerable interés internacional en emplearlos. Como reporta Seidler (2006), los ingenieros son muy demandados en China, mientras que los médicos y también los trabajadores de educación social se dirigen al Reino Unido. Los alemanes que se desempeñan como trabajadores calificados de la construcción tienen 
mucha demanda en casi todas partes, dentro de Europa, desde Noruega hasta Suiza y España, así como en los países más lejanos como Australia y Canadá (Beunink 2005).

Seidler enfatiza que, en la mayoría de los casos, para quienes ya tienen empleo en Alemania, el salario no marca una diferencia significativa cuando deciden buscar un empleo en el extranjero. Sus principales motivaciones parecen en cambio las de encontrar mejores condiciones de trabajo, como un empleo seguro, perspectivas favorables a futuro, desarrollo personal y horas fijas de trabajo, siendo este último argumento particularmente importante en el sector salud (Seidler, 2006). Se informa que los científicos jóvenes se ven particularmente atraídos por la atmósfera de investigación más cómoda en Estados Unidos, así como por la mejor dotación financiera de las instituciones de ese país (Biestmann, 2005). Sólo hasta hace poco tiempo se empezó a discutir seriamente sobre la mejor manera de abordar estas cuestiones y, sobretodo, qué medidas adoptar para evitar la fuga de cerebros de Alemania.

\section{REFLEXIONES SOBRE COSTO-BENEFICIO}

Al considerarse a la luz de la teoría económica neoclásica, se esperaría que la creciente emigración trabajara en la dirección de un nuevo equilibrio del mercado de trabajo, caracterizado por menos desempleo y salarios más altos en la región de origen y, por tanto, desembocara en una mejora del bienestar en ese espacio. No obstante, en el mundo real, los beneficios para el país de migración en su conjunto no quedan tan claros porque no se cumplen las suposiciones neoclásicas de una fuerza laboral homogénea - la emigración es más bien positivamente selectiva- y porque los movimientos migratorios casi de manera inevitable involucran movimientos del capital con implicaciones económicas significativas. Una mirada más en detalle de la composición de la población migrante, sus patrones de movilidad y su comportamiento en el renglón de remesas revela un cuadro complejo.

\section{Procedimiento y metodología}

Los costos y beneficios que se asocian con una economía de emigración de su fuerza laboral están compuestos por a) los costos de oportunidad de esa fuerza de trabajo, es decir, el valor de producción que se pierde debido a su salida; $b$ ) el valor de sus remesas en la economía doméstica, y $c$ ) el capital humano adicional adquirido en el extranjero y que traen a casa quienes retornan. Según los principios de un análisis de costo beneficio, con el objeto de asesorar su valor para la sociedad de en el país de origen, estos flujos deben evaluarse respectivamente por sus costos de oportunidad y la utilidad que proporcionan por las metas adicionales que aportan, medidos por la disposición a pagar por ellos. ${ }^{8}$

\footnotetext{
${ }^{8}$ Una adecuada base metodológica para le evaluación es un modelo de tres estapas que ha sido aplicado por Knerr
} 
En los párrafos que siguen se evalúan estos costos y beneficios a partir de una burda base de datos para diferentes grupos de emigrantes. A falta de estadísticas oficiales sobre los determinantes esenciales de los costos y beneficios relevantes, se hacen los cálculos utilizando información y suposiciones en parte esquemáticas pero verosímiles. La Oficina Federal Alemana para la Migración y los Refugiados (Bundesamt für Migration und Flüchtlinge) ofrece anualmente datos oficiales acerca del número de emigrantes, su composición por sexo y edad, su nacionalidad y su destino; empero, no se ofrecen datos acerca de la capacitación de los migrantes, sus profesiones o motivaciones. La información sobre los flujos de remesas de los trabajadores la aporta el Banco del gobierno federal de Alemania (Deutsche Bundesbank). Se explicarán las estimaciones utilizadas cuando aparezcan en la siguiente exposición. El aspecto de la migración de retorno no se considera por separado. ${ }^{9}$

En concordancia con los principios generales del ACB, en los siguiente párrafos suponemos que los emigrantes no son parte de la sociedad alemana mientras estén fuera del país - es decir, los tratamos como extranjeros económicos-. Por lo tanto, el ingreso devengado en el extranjero no se trata como un beneficio para Alemania. En consecuencia, no restamos los beneficios que obtienen los extranjeros que viven en Alemania.

\section{Antecedentes de los datos}

No hay información estadística oficial acerca del perfil profesional, educativo o de capacitación de los emigrantes. Uebelmesser (2006) ofrece algunos datos crudos a partir de su procesamiento de datos del panel socioeconómico alemán (German Socio-Economic Panel - GSOEP), una encuesta en hogares realizada en varias oleadas y que incluye preguntas acerca de las intenciones de migrar al extranjero clasificadas por edad, sexo y escolaridad. Los resultados muestran que la tendencia a emigrar aumenta con la educación, lo que puede resultar sorprendente es el hecho de que los hombres y mujeres con empleo tienen una propensión mucho mayor a emigrar que quienes están desempleados. En total, el 54\% de los que tenían empleo estaban listos para el traslado, mientras sólo el $46 \%$ de los desempleados estaban dispuestos a salir del país. Además, la disposición para emplearse en el extranjero aumenta con el nivel escolar. De tal modo, mientras que el $46 \%$ de los hombres que sólo contaban con educación elemental declaraba que «migrarían fácilmente»o «migrarían si fuera necesario», esta proporción alcanzó el 61\% entre quienes tenían un grado universitario. De acuerdo con Burda et al. (1998), al suponer que las intenciones son una función monotónica de las variables que motivan la migración, puede concluirse que los grupos que expresan una disposición mayor a emigrar también están desproporcionadamente representados entre los migrantes reales.

y Zaqqa (2004), y Zaqqa (2006) para evaluar la exportación de mano de obra desde Jordania, y para revisar si la inversión en capital, por parte del estado, es rentable en el caso de quienes emigraron al extranjero posteriormente.

${ }^{9}$ El riesgo y la incertidumbre asociados con las actividades no se considera, debido a que no hay información disponible sobre este aspecto. 
Según Klaus Bade, miembro del Consejo Alemán de Migración, Alemania pierde cada vez más en la competencia internacional por la pérdida de personal de alto potencial (Bade, 2005). Un número creciente de expertos, en especial del sector de las tecnologías de la información, por ejemplo, salen de Alemania para siempre. Según lo plantea Bade (2005), la situación dentro de las universidades alemanas, que hacen que las carreras académicas sean difíciles y largas, es la causa de que los jóvenes científicos busquen una vocación en el extranjero. ${ }^{10}$

\section{Pérdida de fuerza de trabajo: costos de oportunidad de la fuerza de trabajo alemana}

En 2004, 150,667 alemanes salieron oficialmente de Alemania para establecerse en el extranjero. Al substraer a quienes tenían menos de 18 años de edad y más de 65, que en conjunto representaron el $14 \%$ del total, quedan 129,573 personas en la fuerza de trabajo. Suponiendo lo siguiente:

- los salarios reflejan el costo marginal de oportunidad de la mano de obra;

- quienes tienen un alto nivel educativo pertenecen al grupo de altos ingresos, que los trabajadores manuales calificados al grupo de ingresos medios, y los trabajadores de escasa calificación pertenezcan al grupo de bajos ingresos;

- los emigrantes muestran la misma composición de calificación que el resto de la población; y

- 33\% de los trabajadores de escasa calificación han estado desempleados y, por ende, no implican costos de oportunidad para la economía cuando se van del país. ${ }^{11}$

Con base en estas suposiciones, con el uso de los datos de GSOEP para analizar el comportamiento migratorio de los diferentes grupos de ingreso, llegamos a los resultados que se muestran en la tabla 3, que enumera los costos de oportunidad de la fuerza de trabajo que emigra de Alemania. Demuestran que, bajo las suposiciones mencionadas, Alemania perdió mil quinientos millones de Euros por año, tan sólo por la pérdida de fuerza de trabajo en el año 2004.

\footnotetext{
${ }^{10}$ Además, varios científicos alemanes del más alto nivel se establecieron en Estados Unidos, como el ganador alemán del premio Nóbel, Wolfgang Ketterle, quien es director de un área en la Unión Americana. Además, en 2005, había 10,000 alemanes estudiando en Estados Unidos (Biestermann, 2005). Muchos de ellos se quedan en el extranjero después de terminar sus estudios.

${ }^{11}$ La situación del mercado de trabajo entre Alemania occidental y Alemania oriental (la ex-«República Democrática Alemana») muestra diferencias significativas. Las tasas de desempleo, en particular dentro del grupo de los trabajadores de escasa capacitación, difieren fuertemente entre las dos partes del país. Lo contrario se aplica para algunas profesiones altamente calificadas, como los médicos. Como consecuencia, surgen, en promedio, diferentes costos de oportunidad, según sea el lugar del que proceden los migrantes. Además, nuestros cálculos no toman en cuenta el hecho de que una gran parte de los desempleados ocasionalmente trabaja en la economía informal.
} 
TABLA 3

Costos de oportunidad anuales para la fuerza de trabajo emigrante (en €)

\begin{tabular}{|c|c|c|c|}
\hline CAPACITACIÓN & $\begin{array}{c}\text { NÚMERO, 'OOO } \\
(\text { UNIDADES DE MIGRACIÓN) }\end{array}$ & $\begin{array}{l}\text { SUPUESTO INGRESO BRUTO } \\
\text { ANUAL POR U.M. }{ }^{\mathrm{x}} €\end{array}$ & $\begin{array}{c}\text { COSTO DE OPORTUNIDAD } \\
\text { TOTAL, } € \text { AL AÑO }\end{array}$ \\
\hline Baja capacitación & 29,543 & $\left(2,507^{*}\right) 30,084$ & 59 '5476,980 \\
\hline Calificados & 61,288 & $\left(3,384 €^{* *}\right) 40,608$ & 2'488,783,104 \\
\hline Altamente calificados & 38,742 & $\left(5,000 €^{* * *}\right) 60,000$ & $2^{\prime} 324,520,000$ \\
\hline Total & 129,573 & 130,692 & $5^{\prime} 408,780,084$ \\
\hline
\end{tabular}

* Ingreso bruto promedio al mes de un trabajador en la industria manufacturera. ${ }^{* *}$ Ingreso bruto promedio mensual de un empleado en la industria, el comercio, el crédito o los seguros.

${ }^{* * *}$ Suposición basada en diversas fuentes de información. ${ }^{* * * *} 88,8771,612 \times 0.67$

x Unidad de migración= porción de la fuerza de trabajo (según el nivel de ingreso) $\mathrm{x}$ (propensión a migrar de ese grupo), calculado con datos de GSOEP, cit. por Uebelmesser, 2006.

Fuente: cálculos de la autora con base en datos de la Statistisches Bundesamt, la encuesta de GSOEP y Uebelmesser (2006).

Dado que - según los datos de la encuesta de GSOEP - la propensión a migrar se incrementa con el nivel de educación y se reduce con la edad (Uebelmesser, 2006), Alemania pierde de manera desproporcionada una fuerza de trabajo joven altamente calificada.

La emigración de jóvenes científicos puede implicar una pérdida particularmente grande de capital humano en el largo plazo, porque todavía les queda por recorrer una gran parte de su vida productiva. Cada año, cerca de 50,000 jóvenes científicos estudia en el extranjero. Se calcula que uno de cada siete estudiantes de doctorado sale de Alemania, la mayoría hacia Estados Unidos. Según la Asociación de Académicos Alemanes (German Scholars Association - GSo) en 2005, cerca de 6,000 jóvenes científicos trabajaban en Estados Unidos y, de ellos, una cuarta parte no regresó a Alemania (Biestmann, 2005). Muchos se fueron a vivir a Suiza, en donde, en conjunto, el $22 \%$ del personal de las universidades proviene de Alemania ${ }^{12}$ (Hahne 2005). Sólo una minoría regresa. ${ }^{13}$

\section{Costos de oportunidad de la fuerza de trabajo extranjera}

La emigración de los extranjeros - y su respectiva y reducida inmigración - también implica costos y beneficios para la economía alemana. No hay suficiente información dispo-

\footnotetext{
${ }^{12}$ Una tercera parte del personal de las universidades en Suiza proviene del extranjero, dos terceras partes de éste se hallan compuestas por alemanes.

${ }^{13}$ Puede ser que también haya ciertas pérdidas de capital humano por la emigración de los pensionados, porque
} 
nible acerca de las credenciales de quienes se van y que pudiera utilizarse para calcular el valor del capital humano que Alemania pierde de ese modo. No obstante, la composición nacional de los migrantes sugiere que la emigración está sesgada selectivamente hacia los extranjeros con niveles de escolaridad altos. De tal modo, en 2004 el saldo migratorio con los ciudadanos de los estados de la UE-14 era negativo, con $-33,817$ mientras que era positivo con $+34,634$ para los ciudadanos de los 10 nuevos miembros de la UE, es decir, con los provenientes de Europa central y del este que se unieron a la uE en 2004 y que, en promedio, tienen un nivel escolar más bajo (Statistisches Bundesamt, 2006 y Banco Mundial).

Al comparar la emigración con la inmigración, resulta esclarecedor ver que hay una creciente emigración neta de parte de quienes provienen de países económicamente más desarrollados y una inmigración neta de parte de las personas que vienen de países menos desarrollados, es decir, esencialmente los llamados países en desarrollo y los países de Europa central y del este. De tal modo, la migración neta respecto al continente africano, que muestra un nivel de educación por debajo del promedio, se incrementa desproporcionadamente con el tiempo (véanse los datos del Statistisches Bundesamt, 2006 y el Banco Mundial). El saldo migratorio tiende, pues, a estar sesgado hacia el lado negativo en lo que respecta a los nacionales de países con un nivel educativo más alto y hacia el lado positivo en el caso de quienes proceden de países en los que el nivel educativo es más bajo (véase fig. 6).

FIGURA 6

Inmigración y emigración desde y hacia Alemania por nacionalidades

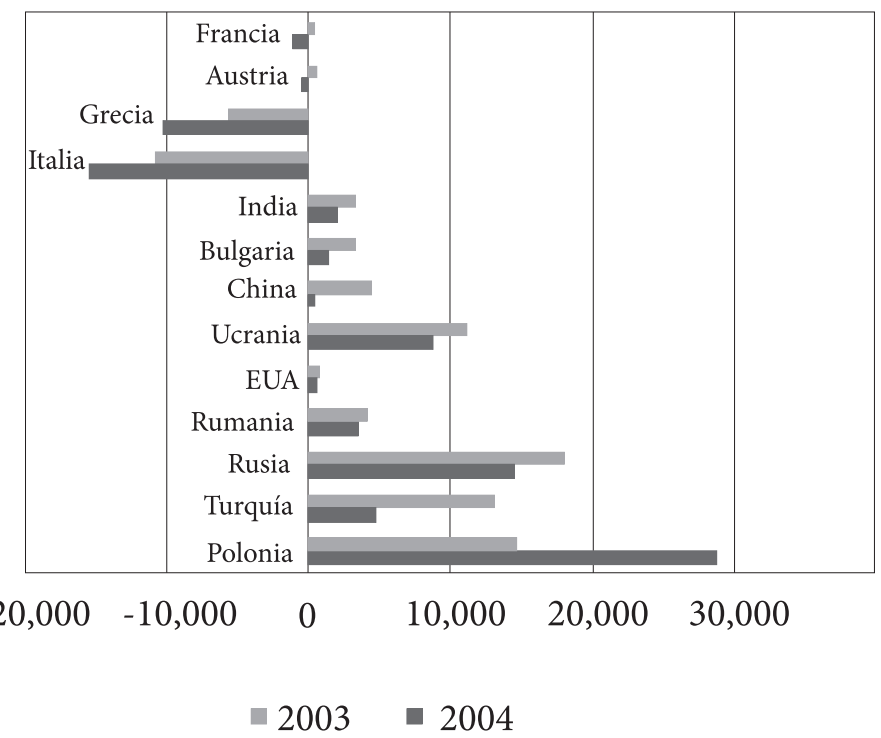

Fuente: basado en datos de la Statistisches Bundesamt.

muchos de quienes pasan su jubilación en el sur de Europa han elegido la jubilación adelantada y podrían haber sido sustituidos por personal con menos experiencia. 
Los extranjeros que salen del país exportan capital humano que podría ser útil para la economía de Alemania. Esto se aplica a lo que trajeron consigo al llegar como personas calificadas y con alta escolaridad, así como a lo adquirido al trabajar y vivir en Alemania. El último componente es específico del país y podría ser particularmente valioso para su economía. Los países a los que se dirigen se benefician con estos recursos.

Además, la inmigración en descenso implica costos y beneficios. No se distribuye de igual manera de un nivel de calificación al siguiente y, quizá, la cantidad de personas calificadas desciende más en términos proporcionales que la cantidad correspondiente para los menos calificados. De tal modo, por ejemplo, la cifra de los especialistas en tecnología de la información que inmigraron disminuyó a la mitad desde principios a mediados de la primera década del siglo xxi (Bundesamt für Migration und Flüchtlinge, 2006). Al mismo tiempo, Alemania enfrenta una creciente escasez de mano de obra altamente calificada y un exceso en la oferta de mano de obra escasamente calificada o de nula capacitación. La mayor parte de los inmigrantes, trabajadores temporales que se ocupan varias semanas o meses en la agricultura y que provienen principalmente de Polonia, constituyen un grupo numéricamente estable de cerca de 250,000, ya que su empleo está sujeto a una cota (véase Pruin y Knerr, 2006).

Considerando el hecho de que en 2005 había casi el mismo número de migrantes que entraban en el país como de los que salían de él (Stat. Bundesamt, 2006), a primera vista parece haber una migración de reemplazo. Mas, en el desarrollo económico del país, la inmigración y la emigración no producen un equilibrio, ya que hay una disparidad significativa entre las credenciales de los inmigrantes y las cualidades de los emigrantes. Parece posible que esos extranjeros con mejores oportunidades de empleo, es decir, los más calificados salgan de Alemania mientras que se aplica lo contrario a los menos calificados y a los desempleados que tienen derecho a beneficios de desempleo y otros más dentro de Alemania. En la misma categoría de habilidades formales, el capital humano específico de países particulares se va del país mientras que los más inexpertos entran en él.

\section{Remesas}

Asociadas con la emigración, las remesas pueden ser benéficas para Alemania, ya sea que los recursos que representan sean enviados o transportados por la mano de obra en el extranjero, por lo que los beneficios de quienes no reciben remesas dependen de los comportamientos de gasto de los receptores, así como de quienes gastan el dinero en el segundo ciclo y los subsecuentes. Ello se refleja en el multiplicador de gasto macroeconómico que a mediados de la primera década del siglo, se calcula, es de 0.63 en Alemania (Deutsche Bank, 2005).

\section{Remesas recibidas}

No hay información disponible acerca de la magnitud de las remesas que envían los 
alemanes desde el extranjero. Sin embargo, no son despreciables, ya que una parte significativa de las personas de altos potenciales no trabajan en el extranjero de manera permanente, sino que conservan su lugar de vivienda en Alemania. Esto se aplica, por ejemplo, a muchos de los médicos que trabajan en Gran Bretaña, aunque no hay datos estadísticos disponibles acerca de su magnitud. ${ }^{14}$ Las estimaciones acerca de la cantidad transferida serían simples conjeturas. ${ }^{15}$

Se pueden sacar algunas conclusiones del desarrollo de la emigración de los alemanes. A medida que aumenta el número de emigrantes, deberían aumentar también sus remesas. Se reportan casos frecuentes de médicos que trabajan en Gran Bretaña durante los fines de semana o vacaciones. Cabría esperar que transfirieran la mayor parte de sus ganancias a Alemania, ya sea por transferencia bancaria o en propia mano. Lo mismo se aplica a quienes trabajan en el extranjero por temporadas, cosa que es más frecuente en el sector del turismo. Por otro lado, hay grupos de migrantes de los que no podría esperarse que enviaran cantidades significativas. De tal modo, los jóvenes científicos difícilmente envían dinero a Alemania. Por el contrario, una parte considerable de ellos estudia en el extranjero con una beca del gobierno alemán, lo que implica una salida de dinero del país.

La amplia categoría de transferencias privadas, pagos desde el extranjero, pensiones y pagos de sostenimiento incluye las remesas de la mano de obra que llegan desde el extranjero, pero éstas no juegan un papel significativo. Además, las transferencias de menos de 12,500 € no se reportan, aun cuando lo más probable es que la mayor parte de los envíos sean de montos menores a ese límite.

El fuerte impacto económico de las remesas ha sido demostrado por diversos investigadores (Knerr, 1994; Gallina, 2006). No obstante, estos resultados difícilmente pueden generalizarse al caso de Alemania, porque esas investigaciones se refieren al efecto que tienen estos recursos en los países de bajos ingresos. El patrón típico de gasto de las remesas es diferente en un país industrializado como Alemania. Aún cuando no hay información acerca de los usos a que se destina el dinero, se puede asumir con cierto grado de certeza que se gasta de acuerdo con el anterior patrón de gasto de los migrantes y de sus familias porque, por lo regular, no hay gran diferencia en el ingreso de esas familias. Suponiendo que las remesas se gastan como cualquier otro dinero, el

\footnotetext{
${ }^{14}$ Las remesas de los trabajadores no se registran por separado en la balanza de pagos de Alemania (Deutsche Bundesbank, 2006b). «No hay una categoría que corresponda a las remesas al extranjero de parte de los trabajadores inmigrantes y que reflejaría las transferencias hacia Alemania. Los pagos que ingresan que corresponderían a esa categoría se incluyen en la categoría 'transferencias privadas, pagos desde el extranjero, pensiones y pagos de sostenimiento'. Sin embargo, hay que observar que la mayor parte de esos pagos muy probablemente debe estar debajo del límite de las cantidades que es obligatorio reportar y por lo tanto no puede incluirse en la balanza de pagos. Además, debido a la falta fuentes estadísticas no es posible realizar procedimientos de estimación para esas categorías» (Deutsche Bundesbank, 2006b, traducido aquí al español de la traducción del alemán al inglés por la autora).

${ }^{15}$ No obstante, el Banco Mundial ofrece información acerca del monto de las remesas que llegan a Alemania bajo el encabezado de «remesas recibidas de los trabajadores alemanes y compensación de los empleados». Muestran una cantidad de casi $\$ 7$ mil millones para 2004. Lo que no parece factible dados los antecedentes de la información disponible que ofrece el Deutsche Bundesbank y acerca de la magnitud y estructura de la emigración de alemanes. Para detalles véase el anexo I.
} 
multiplicador sería de 0.62 (Deutsche Bank research 2005). Las remesas que entran al país superan por un ligero margen a las que salen. En buena medida debido a las diferencias entre las bases de datos proporcionadas, por un lado, por el servicio alemán de estadística y la información que provee el Banco Mundial, por el otro (véase nota 15), no se justifica una comparación directa entre ambos.

\section{Remesas enviadas al extranjero}

Desde principios de los años noventa, se ha observado una caída significativa y creciente en las remesas que se envían desde Alemania (véase fig. 7). A medida que desciende la cantidad de inmigrantes, así disminuyen las remesas al exterior. Otra razón para el descenso puede ser la situación económica cada vez más insegura de los migrantes, resultado de lo cual pueden ser los ingresos más bajos, así como una propensión menor a enviar dinero a los familiares en el extranjero. Además, debido a la reducción de la inmigración, la proporción de las familias migrantes establecidas aumenta y éstas ven cada vez más el centro de su vida y de su futuro en Alemania, y cada vez menos en su país de origen. La inmigración decreciente de la fuerza de trabajo, la emigración de los extranjeros, combinadas con el desempleo, han desembocado en una caída en el flujo de remesas desde Alemania. Tras un nivel máximo en 1983, posteriormente han descendido de manera permanente

FIGURA 7

Remesas enviadas desde Alemania, 1971-2006

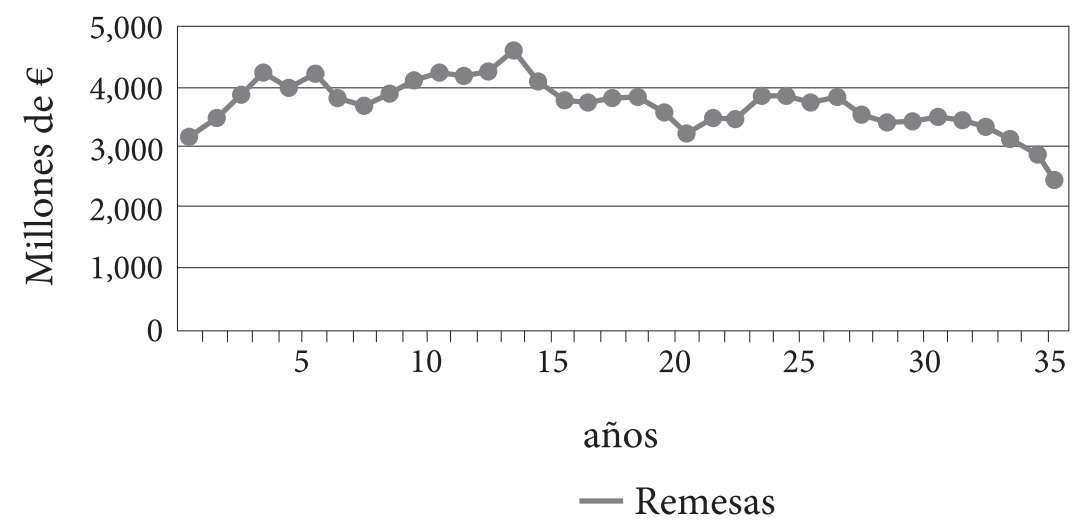

Fuente: con base en datos del Deutsche Bundesbank, 2006.

Un cierto porcentaje de los emigrantes son pensionados que gustan de pasar su tiempo en las regiones cálidas y soleadas del sur de Europa, sobre todo en España. Aún cuando sus decisiones de migrar no parecen estar influidas directamente por el desarrollo económico de Alemania, su salida tiene consecuencias económicas dado que involucra la salida de capital y poder adquisitivo que, en adelante, ya no estimulará a 
la economía alemana sino a los correspondientes al país de destino. Los países que se benefician de los pensionados alemanes son sobre todo España y, aunque en mucho menor medida, Turquía.

\section{Efectos distributivos}

La migración y las remesas tienen implicaciones distributivas que hacen que el conjunto total quede exento de cambios y, por lo tanto, no se considerarán en un análisis puro de costo-beneficio. No obstante, pueden tener importantes repercusiones en la sociedad y en el desarrollo económico a largo plazo. Un efecto de importancia en este contexto es el de los ahorros de los beneficios del desempleo, que quedan disponibles para ser usados con otros propósitos a partir del momento en que los desempleados salen del país. Es decir, el 33\% de los alemanes que buscan empleo en el extranjero dentro de ZAV están desempleados al momento de hacerlo. Si encuentran puestos de trabajo en el extranjero, la hacienda pública ahorra los recursos que utilizaba previamente para sostenerlos. Con un beneficio mensual por desempleo de aproximadamente $705 €,{ }^{16}$ ello equivale a $8,460 €$ anuales por persona. Suponiendo un total de 8,863 desempleados que se van del país, según se calculó más arriba, esto equivale a $€ 74$ '980,980 anuales.

\section{Implicaciones a largo plazo}

Los procesos relevantes para la estructura y el tamaño de la población se desenvuelven lentamente. «Los cambios en los componentes esenciales del desarrollo de la población - tasas de nacimiento, mortalidad y migración - por lo regular muestran todo su impacto en la situación de la población después de varias décadas» (Statistisches Bundesamt, 2003: 9).

\section{POSIBLE REFLUJO DEL CAPITAL HUMANO}

La emigración de las personas con altos potenciales podría enriquecer a Alemania en el largo plazo si aumentan su conocimiento y experiencia mientras están en el extranjero y regresan después de un cierto tiempo. La información acerca de qué tanto se aplica esta afirmación es apenas esquemática. Se puede suponer que tres cuartas partes de los jóvenes científicos que siguen su educación en Estados Unidos y regresan a Alemania con el tiempo fuera de país han mejorado su capital humano. La conciencia de los alemanes en este renglón, tanto de los diseñadores de política como del público, va en aumento.

\footnotetext{
${ }^{16}$ Calculado a partir de la suma de $345 €$ mensuales para gastos de sostenimiento y $360 €$ para la renta de una vivienda, a la que el desempleado tiene derecho de acuerdo con la ley alemana («Arbeitslosengeld II»). Bundesgesetzblatt, $30^{\text {th }}$ March 2006.
} 


\section{La cambiante composición por edad y capacitación de la fuerza de trabajo alemana}

Stephanie Wahl, directora administrativa del Instituto de Investigación de la Economía y la Sociedad (IWG) ${ }^{17}$ afirma que el problema a principios del siglo xxi no es tanto el grado de emigración, sino la creciente propensión de los alemanes jóvenes con alta escolaridad a dejar el país, lo que podría ser un sinónimo del bajo atractivo de Alemania. Además, agrega que «quienes se van, están altamente motivados y bien entrenados y educados. Quienes llegan, en la mayoría de los casos son pobres, carecen de capacitación y tienen baja escolaridad» ${ }^{18}$ (Wahl, citado por Hahne, 2005).

Klaus Bade describe la situación como sigue: «Alemania se encuentra en una situación migratoria suicida porque cada vez somos menos capaces de ofrecer una perspectiva a la gente joven y competente, mientras que, en su mayor parte quienes inmigran están escasamente calificados [...] por lo tanto, perdemos nuestra sangre» (Bade en Müller, 2006, traducido del alemán al inglés por la autora).

La desproporcionada emigración de alemanes con alto potencial puede reforzar las tendencias ya existentes hacia los cambios en las estructuras poblacionales: dentro de la decreciente población alemana, con una tasa de nacimiento de 1.3 niños por mujer, los inmigrantes que muestran tasas de nacimiento más altas que el grupo de alemanes con la mejor calificación laboral, haría que la proporción de habitantes con antecedentes migratorios se incrementará aún más, así como los hijos de las familias con menor educación (véase Kaufmann, 2005).

\section{Implicaciones para \\ el sistema de pensiones}

Las implicaciones de la emigración de los más jóvenes en edad de trabajar se sentirán en el sistema de pensiones del país, ya que los emigrantes dejan de aportar al sistema. Alemania se ha topado con una paradoja poblacional ya que mientras mantiene un desarrollo económico creciente, los parámetros de vida material y las tasas de nacimiento disminuyen (Birg, 2004). Una paradoja adicional es el efecto del trinquete, es decir, esta tendencia no se revierte en épocas de crisis económica, sino que parece fortalecerse: según una encuesta del Instituto Federal Alemán de Investigación en Población, ${ }^{19}$ los jóvenes en Alemania se cohíben de tener hijos debido a la inseguridad económica.

La emigración de los jóvenes refuerza las consecuencias de una composición de edad desfavorable y agrava aún más la situación, en particular en el sistema de pensio-

\footnotetext{
${ }^{17}$ Institut für Wirtschaft und Gesellschaft (IWG).

${ }_{18}$ Traducción de la autora del original alemán al inglés.

${ }^{19}$ Bundesinstitut für Bevölkerungsforschung. 
nes, pero también en el sistema de salud, pues el número de quienes pagan por estos decae todavía más. Esto podría producir un círculo vicioso: la gente joven y sana se va del país, una de las razones de ello es el hecho de que, al comparar ingresos netos, tienen que pagar grandes cantidades de dinero al sistema social - sistema de pensiones, seguro de salud, seguro de desempleo-. A medida que se incrementa aún más el coeficiente de dependencia, la carga para quienes se quedan se incrementa. Esto podría, a su vez, promover la emigración de otros.

Las tasas de nacimiento en declive en Alemania desembocan en razones menores de dependencia, lo que en el otro extremo de la escala de edad se contrabalancea por una creciente expectativa de vida $\mathrm{d}=\left(\mathrm{P}_{0-20}+\mathrm{P}_{65}\right)\left(\mathrm{P}_{20-64}\right)^{-1}$ (Dinkel, 1989:251). Respecto a las conclusiones que pueden derivarse de la carga de la población trabajadora, sin embargo, la fórmula resulta engañosa. Existe - con la misma (d - a - - una diferencia entre la razón de dependencia causada por una proporción mayor de jóvenes y una causada por una proporción mayor de gente de edad avanzada. La emigración de las partes más jóvenes de la población deja al resto con más gente vieja en términos comparativos, mientras que en muchos casos los hijos - si los migrantes los tienen - son llevados con los migrantes al extranjero o habrán de nacer allá.

\section{Desequilibrios \\ entre los géneros}

La migración hacia y desde Alemania es un fenómeno predominantemente masculino. De ahí que traiga consigo un desequilibrio en la estructura de la población. Aún cuando la emigración desde Alemania no constituye un fenómeno masivo, pueden manifestarse algunos efectos marginales. Con 62.5\% en 2004, la porción de los varones emigrantes era superior a la de los inmigrantes, en donde alcanzaba apenas el 58.4\%. Ambas cifras se han incrementado permanentemente a lo largo de las décadas anteriores; en 1991 alcanzaban el 57.1 y el 55.7\%, respectivamente (Bundesamt für Migration und Flüchtlinge, 2006: 139). Como consecuencia, la fuerza laboral femenina podría ocupar una porción cada vez mayor del segmento de mercado de trabajo de los altamente calificados.

\section{Formación de redes}

Como en muchos otros países, el goteo de la emigración puede convertirse en un río promovido por las redes de amigos y parientes, lo que eventualmente podría ser un fenómeno que se sostenga por sí mismo y, en parte, llegar a ser independiente del desarrollo económico de Alemania. También debido a esos efectos, «el remolino se ha desatado y no cejará en un periodo breve de tiempo» (Bade en Müller, 2006, traducción del alemán al inglés por la autora). El flujo de la emigración podría incluso centrarse más específicamente en los profesionistas, a través del carácter específico de las redes privadas (Knerr, 1994). 


\section{CONCLUSIONES}

Alemania ha evolucionado de ser un país clásico de inmigración a uno de emigración. Mientras que los países de la UE, que por décadas se han caracterizado por una emigración neta, se han convertido en países de inmigración, Alemania recorre el camino en el otro sentido. Aunque, al medirse por la proporción de la fuerza laboral total, la emigración de la fuerza de trabajo alemana todavía se presenta como insignificante, su composición y perspectivas parecen plantear cada vez más una amenaza a la economía alemana. Aún cuando hay cierto alivio en la hacienda pública por medio de la partida de los desempleados, en su mayoría escasamente calificados, quienes tienen altos potenciales, cada vez más escasos en la economía alemana, muestran una propensión creciente a migrar.

Además, la experiencia internacional sugiere que, debido a la formación de redes, el goteo de la migración puede convertirse rápidamente en un río. Dado que la composición de la fuerza de trabajo que inmigra no corresponde con los requerimientos de la economía alemana, es de esperar que, en el futuro cercano, sectores clave se enfrenten con una escasez de mano de obra calificada. Los resultados de estas reflexiones tienen un conjunto de implicaciones para una política migratoria al poner el desarrollo económico en el centro de la reflexión. No obstante, para elaborar políticas adecuadas, se requiere una base más amplia de datos y conocimiento de las relaciones. Promover la investigación en esa dirección podría ser el próximo paso a dar.

A la luz de las bajas tasas de nacimiento del país y de la desproporcionada emigración de la mano de obra altamente calificada, la inmigración de personas con altos potenciales podría tener resultados positivos para el desarrollo económico alemán. Por lo tanto, sería benéfica una mejoría en la composición de la inmigración. Una posibilidad para lograr esa meta sería un sistema de puntajes para seleccionar a los inmigrantes, similar al aplicado en Estados Unidos. Además, considerando los aspectos económicos, tendría sentido intentar conservar a los migrantes altamente calificados dentro del país, aún cuando hubiesen ingresado con un status que inicialmente no los autorizara a quedarse para trabajar. Al mismo tiempo, deberían de promoverse esfuerzos de mediación para que los desempleados consigan puestos de trabajo en el extranjero.

Es necesario establecer esfuerzos más enérgicos para atraer a los jóvenes científicos de regreso al país, después de algunos años valiosos que han pasado en su preparación en el extranjero. No se trata sólo de salarios, sino que ello también esté determinado por la atmósfera en las universidades y las instituciones de investigación, lo que podría llevar a desarrollar y poner en práctica ideas innovadoras. El gobierno alemán gasta grandes sumas de dinero en becas para enviar a personas a estudiar al extranjero y acumular un valioso capital humano. Sin embargo, no se preocupa lo suficiente por su regreso. Ello también tiene consecuencias negativas en el largo plazo para el capital humano en Alemania. ${ }^{20}$

\footnotetext{
${ }^{20}$ Véase también Eicke Weber, Presidente de la organización de académicos alemanes en Estados Unidos, citado por Hahne, 2006.
} 
El paso más importante para conservar la fuerza de trabajo altamente calificada dentro del país sería reforzar el crecimiento económico de Alemania y proporcionar un panorama más optimista a la fuerza de trabajo. Las reflexiones acerca de incentivos especiales para conservar los altos potenciales del país dentro de las fronteras podrían, entonces, convertirse en obsoletas. Sin embargo, su escasez podría haberse constituido ya en una limitación para comenzar el nuevo ímpetu.

\section{FIGURA 8}

Remesas de trabajadores en Alemania y compensaciones recibidas por los empleados en dólares de EUA

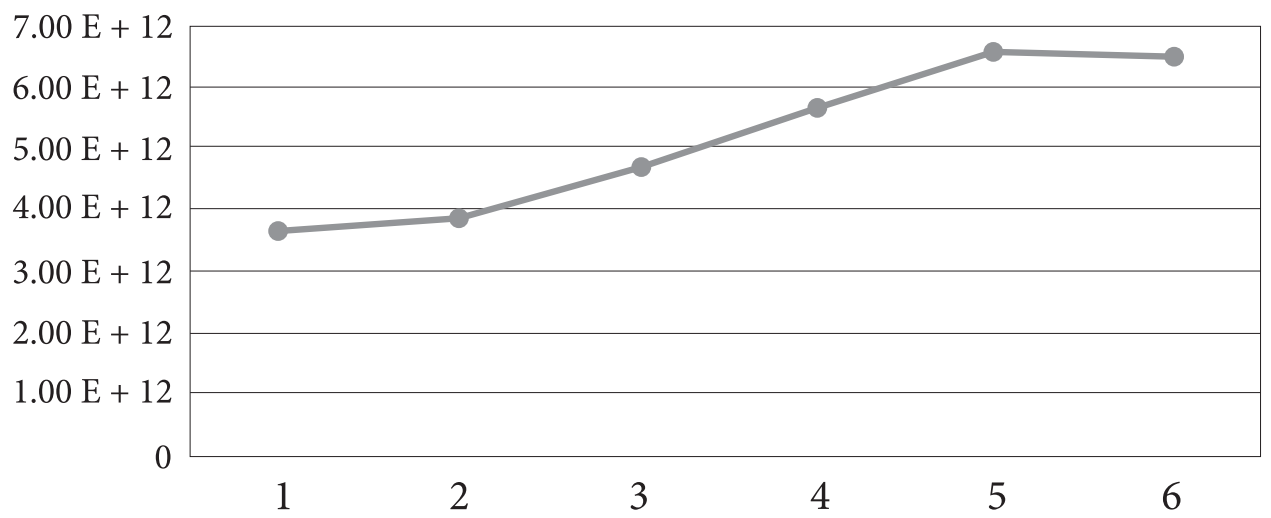

Fuente: con base en datos del Banco Mundial

\section{BIBLIOGRAFÍA}

BADE, Klaus (2005), Entrevista en Frankfurter Rundschau 2.1, 2006.

BEUnink, Ralf, Alpenländer besonders beliebt, Manager-magazin, http://www.manager-magazin.de/koepfe/karriere/0,2828,388527,00.html.

Biestmann, Stefan (2005), Abwanderung ohne Ende, Manager-magazin.

BIRG, Herwig (2004b), «Ergebnisse international vergleichender Forschung», en Bundeszentrale für politische Bildung (Hg.), Bevölkerungsentwicklung, Munich, Franzis’ print \& media GmbH, S., pp. 11-13.

Bundesamt Für Migration und FlüChtlinge (2006), Migrationsbericht im Auftrag der Bundesregierung (Migrationsbericht 2005), Berlín.

Bundesgesetzblatt, 30 de marzo de 2006.

Bundesinstitut für Bevölkerungsforschung und Robert-Bosch-STIfTung (2006), Generation and Gender Survey, 2005.

Burda, M.C., W. Härdle, M. Müller y A. Werwatz (1998), Semiparametric «Analysis of German East-West migration intentions: facts and history», Journal of Applied Econometrics 13, pp. 531-533.

Castles, Steven (1986), «The guest-worker in Western Europe. An obituary», Inter- 
national Migration Review, Número especial sobre programas para trabajadores temporales: mecanismos, condiciones, consecuencias, vol. 20, pp. 761-778.

Claus, Frieder (2006), Löhne und Wirtschaftswachstum in Deutschland, edición de Diakonisches Werk, Baden Württemberg.

Deutsche Bank Research (2003), «Haushaltskonsolidierung: Wunschdenken und harte Realität», Aktuelle Themen núm. 256 (17/2/2003), Frankfurt.

Deutsche Bundesbank (2006a), Balance of Payment Statistics, Frankfurt.

Deutsche Bundesbank (2006b), Comunicación por correo electrónico con fecha 30/8/2006, firmada por Dirk Mühlhausen

Dinkel, Reiner (1989), Demographie, Bd.1, Bevölkerunsgsdynamik, Munich.

Gallina, Andrea (2006), «Migration, Financial Flows and Development in the EuroMediterranean Area», The Journal of North African Studies vol. 11 (1), pp.17-34.

Hahne, Peter (2005), «Deutschland wird Auswanderungsland. Viele unterbezahlte oder arbeitslose Fachkräfte suchen im Ausland ihr Glück», Boletín Migration und Bevölkerung, edición del 7 de septiembre de 2005.

InTERnational Bank for Reconstruction and Development (Banco Mundial), World Development Indicators, Diversos números.

Kaufmann, Franz-Xaver (2005), Schrumpfende Gesellschaft. Vom Bevölkerungsrückgang und seinen Folgen, Frankfurt del Meno.

Knerr, Béatrice (1997), Labour migration from developing countries. Macro-economic impacts and policy interventions, Kassel.

KNERR, Béatrice y Nadim Zaqqa (2002), Economic Costs and Benefits of Human Capital Migration from Jordan, Paper presented at the first World Congress of Middle East Studies, Mainz.

KöRner, Heiko (1990), Internationale Mobilität der Arbeit, Darmstadt.

MülLER, Henrik (2006a), «Auswanderungswelle 'Wir bluten aus': Entrevista a Klaus Bade», Manager-magazin núm. 7/2006.

MüLLER, Hendrik (2006b), Auswanderungswelle: «Wir bluten aus» manager-magazin und spiegel-online: http://www.manager-magazin.de/unternehmen/artikel/0,28 28,422371,00.html.

Pruin, Anja y Béatrice Knerr (2006 - en prensa), Die Bedeutung ausländischer Saisonarbeitskräfte für verschiedene Wirtschaftssektoren Deutschlands, en: Béatrice Knerr (ed.), Vorweggenommene Erweiterungen: Wanderungsbewegungen aus Grenzgebieten in die EU, Kassel.

Seith, Anne (2006), «Deutsche Auswanderer: Die Tschüss-AG», Spiegel Online 11 $1^{\text {th }}$, August 2006, URL: http://www.spiegel.de/wirtschaft/0,1518.430943,00.html, consultada el 28/8/2006.

Statisches Bundesamt, Statistisches Jahrbuch, Diversos números, Wiesbaden.

Statistisches Bundesamt (2005), Fachserie 1, Wiesbaden.

StraubhaAR, Thomas (1988), On the economics of international labour migration, Berna \& Stuttgart.

Uebelmesser, Silke (2006), «To go or not to go: emigration from Germany», German Economic Review vol. 7 (2), pp. 211-231. 
Wirtschafts- Und Sozialwissenschaftliches Forschungsinstitut der HansBöCKler-STifTung (wsi) (2005), Europäischer Tarifbericht, Bonn.

ZAQQA, Nadim (2006), Economic Development and Export of Human Capital: A Contradiction?, Kassel. 